\title{
SPLIT HOPKINSON PRESSURE BAR: DESIGN PARAMETERS AND PREDICTION OF THE EXPERIMENT OUTPUT
}

\author{
T. Fíla*
}

\begin{abstract}
In this paper, a simple analytic model of Split Hopkinson Pressure Bar (SHPB) propelled by gas-gun is introduced. The model allows for prediction of the output of SHPB experiment or can be used inversely as a design tool for gas-gun propelled SHPB. The model is based on existing models of light gas-gun working according to adiabatic process and on one-dimensional wave propagation theory in linear elastic cylindrical slender bar. The model allows for calculation with elementary drag effects. Model functionality was evaluated and compared with the experimental results of a SHPB setup equipped with linear elastic (aluminium) bars and visco-elastic (polymethyl metacrylate) bars at two different impact velocities.
\end{abstract}

Keywords: Hopkinson bar, SHPB, SHPB design, gas-gun, impact testing

\section{Introduction}

Split Hopkinson Pressure Bar (SHPB) is a well-established experimental technique for testing of materials under high strain-rates. Parameters and performance of the experimental setup have to be tailored for the tested materials as for SHPB with gas-gun the maximal strain and maximal strain-rate achievable in the specimen are very limited. Strain in the specimen is proportional to the wave length of the incident pulse and to its amplitude whereas strain-rate is proportional to the incident pulse amplitude. Thus, these two most important parameters are proportional to the length of the striker bar and its impact velocity. In this paper, a simple analytic prediction model for calculation of the SHPB experiment output is introduced. The model is based on previously published relations dealing with the gas-gun (Rohrbach et al., 2011; Stephenson, 1961; Seigel, 1981) and Hopkinson bar theory (Gallina et al., 2003). Existing models are used to derive modified solution for our design, in which the SHPB striker is propelled using compressed air. Predicted values calculated using this analytic model were compared with the measured values for the experiments conducted with two types of the measurement bars (aluminium and polymethyl metacrylate PMMA) at different strain-rates. Model can be used also inversely for calculation of the required SHPB gas-gun performance needed for desired strain and strain-rate values in the specimen.

\section{Gas-gun and SHPB analytic model}

Literature-based analytic model of gas-gun (Rohrbach et al., 2011; Stephenson, 1961; Seigel, 1981) working according to adiabatic process with drag effects, and analytic model of SHPB based on linear onedimensional wave propagation theory in cylindrical slender bars (Gallina et al., 2003) were used as a basis for the following derivation of the presented model.

\section{Nomenclature}

$\beta \quad$ geometrical relation of the striker and the incident bar $\left(\beta=\frac{A_{\mathrm{st}}}{A_{\text {in }}}\right)$

$$
\begin{array}{ll}
\dot{\epsilon}_{\mathrm{sp}} & \text { specimen strain-rate } \\
\epsilon_{[\mathrm{in}, \mathrm{ref}]} & \text { pulse amplitude (in - incident pulse, ref } \\
& \text { - reflected pulse) } \\
\epsilon_{\mathrm{Sp}} & \text { maximum strain in the specimen }
\end{array}
$$

Tomáš Fíla: Czech Technical University in Prague, Faculty of Transportation Sciences, Konviktská 20; 110 00, Prague; CZ, fila@fd.cvut.cz 
$\gamma \quad$ specific heat ratio ( $\frac{7}{8}$ for compressed air gas-gun)

g gravitational acceleration constant

$\rho$ density of the bars and the striker

$\sigma_{[\text {in,ref,tr,st }]}$ stress (in - incident pulse, ref - re-

flected pulse, tr - transmission pulse, st

- striker bar)

$\sigma_{\mathrm{sp}} \quad$ effective stress in the specimen (ideal plastic material model)

$A_{\text {[sp,st,in,tr] }}$ cross-section area (sp - specimen,

st - striker bar, in - incident bar, tr transmission bar)

$c_{0}$ nominal wave velocity in the bars ( $\sqrt{E / \rho}$ for ideal linear elastic material)
$C_{\mathrm{f}} \quad$ friction coefficient $C_{\mathrm{f}} \geq 0$

$C_{\mathrm{P}} \quad$ pressure loss coefficient $C_{\mathrm{P}} \in\langle 0 ; 1\rangle$

$E \quad$ Young's modulus of the bars

$F_{\mathrm{f}} \quad$ friction force $\left(F_{\mathrm{f}}=m_{\mathrm{st}} \cdot \mathrm{g} \cdot C_{\mathrm{f}}\right)$

$l_{\mathrm{br}_{\mathrm{ef}}} \quad$ barrel effective length $\left(l_{\mathrm{br}}=l_{\mathrm{br}}-l_{\mathrm{st}}\right)$

$l_{\text {br }} \quad$ barrel length

$l_{\text {ps }} \quad$ pulse length

$l_{\mathrm{sp}} \quad$ specimen length

$l_{\text {st }} \quad$ striker bar length

$m_{\text {st }} \quad$ mass of the striker bar

$P \quad$ gas-gun pressure

$t_{\mathrm{ps}} \quad$ pulse duration

$V \quad$ volume of the gas-gun reservoir

$v_{\text {st }} \quad$ striker impact velocity

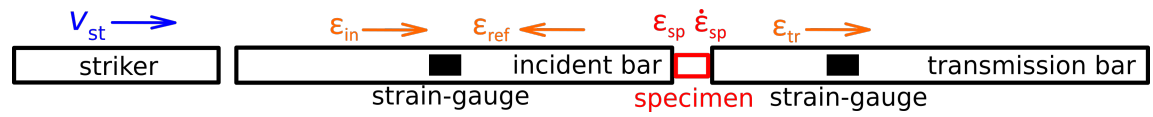

Fig. 1: Scheme of the SHPB principle

Principle of SHPB apparatus is shown in Fig. 1. Based on the adiabatic model for gas-gun, the following equation can be used for the calculation of the striker impact velocity including drag effects represented by the pressure loss coefficient $C_{\mathrm{P}}$ and by the striker bar friction force $F_{\mathrm{f}}$,

$$
v_{\mathrm{st}}=\sqrt{\frac{2}{m_{\mathrm{st}}}\left\{\frac{C_{\mathrm{P}} \cdot P \cdot V}{\gamma-1}\left[1-\left(\frac{V}{A_{\mathrm{st}} \cdot l_{\mathrm{br}_{\mathrm{ef}}}+V}\right)^{(\gamma-1)}\right]-F_{\mathrm{f}} \cdot l_{\mathrm{br}_{\mathrm{ef}}}\right\} .}
$$

From the value of the striker bar velocity $v_{\text {st }}$, the nominal strain-rate in the specimen can be determined according to the relation

$$
\dot{\epsilon}_{\mathrm{sp}}=\frac{2 c_{0}\left(v_{\mathrm{st}} \cdot A_{\text {in }} \cdot \rho \cdot c_{0} \cdot \beta-A_{\mathrm{sp}} \cdot \sigma_{\mathrm{sp}}-A_{\mathrm{sp}} \cdot \sigma_{\mathrm{sp}} \cdot \beta\right)}{A_{\text {in }} \cdot E \cdot l_{\mathrm{sp}}(1+\beta)} .
$$

Parameters of the initial incident pulse and stresses in the bars and in the striker can be derived from the one-dimensional wave propagation theory according to

$$
\begin{aligned}
l_{\mathrm{ps}} & =2 l_{\mathrm{st}} \\
t_{\mathrm{ps}} & =\frac{2 l_{\mathrm{st}}}{c_{0}}, \\
\sigma_{\mathrm{in}} & =\frac{A_{\mathrm{sp}}}{A_{\mathrm{in}}} \sigma_{\mathrm{sp}}+\frac{E \cdot l_{\mathrm{sp}}}{2 c_{0}} \dot{\epsilon}_{\mathrm{sp}}, \\
\sigma_{\mathrm{ref}} & =-\frac{E \cdot l_{\mathrm{sp}}}{2 c_{0}} \dot{\epsilon}_{\mathrm{sp}}, \\
\sigma_{\mathrm{tr}} & =\frac{A_{\mathrm{sp}}}{A_{\mathrm{tr}}} \sigma_{\mathrm{sp}}, \\
\sigma_{\mathrm{st}} & =\frac{\rho \cdot c_{0} \cdot v_{\mathrm{st}}}{1+\beta} .
\end{aligned}
$$

Strain amplitudes of the pulses in the incident bar and maximum strain in the specimen can be derived from the aforementioned equations yielding

$$
\begin{aligned}
\epsilon_{\mathrm{in}} & =\frac{v_{\mathrm{st}}}{2 c_{0}} \\
\epsilon_{\mathrm{ref}} & =-\frac{\dot{\epsilon}_{\mathrm{sp}} \cdot l_{\mathrm{sp}}}{2 c_{0}} \\
\epsilon_{\mathrm{sp}} & =-\frac{2 l_{\mathrm{ps}} \cdot \epsilon_{\mathrm{ref}}}{l_{\mathrm{sp}}}
\end{aligned}
$$




\section{Comparison of the model with experimental results}

\subsection{Experimental setup}

The model was used for a prediction of the output of the real experiments carried out in the DynLab at the author's workplace. The results of the model were compared with the measured values. A modified Kolsky SHPB setup was used for the experiments with both high-strength aluminium alloy bars (EN-AW7075) and PMMA bars at two different impact velocities. In all experiments, the measurement bars with nominal diameter $20 \mathrm{~mm}$ and length $1600 \mathrm{~mm}$ were used. The bars were instrumented using foil-strain gauges connected in Wheatstone half-bridge arrangement in two different configurations (single gauge on each bar, or three gauges on the incident bar and single gauge on the transmission bar). Three striker bars with nominal diameter $20 \mathrm{~mm}$ and lengths $300 \mathrm{~mm}$ (aluminium), $500 \mathrm{~mm}$ (aluminium) and $198 \mathrm{~mm}$ (PMMA) were used in the study. The striker was accelerated using compressed air gas-gun with maximum operating pressure 16 bar. Other details about the used setup and its instrumentation can be found in (Fíla et al., 2017). Experimental setup is shown in Fig. 2-left.

\subsection{Muzzle velocity measurement}

Muzzle velocity of the gas-gun was experimentally measured using photoelectric sensors mounted at the end of the gas-gun barrel. Calculated and measured muzzle velocity of the aluminium striker with length $300 \mathrm{~mm}$ accelerated with the reservoir pressure $1-6$ bar is shown in Fig. 2-center. In the analytic model, no pressure losses and friction were used as these effect were experimentally found negligible for the used striker and pressures. Results of the impact velocity calculated by the model are in very good agreement with the measured values (see Fig. 2-center).
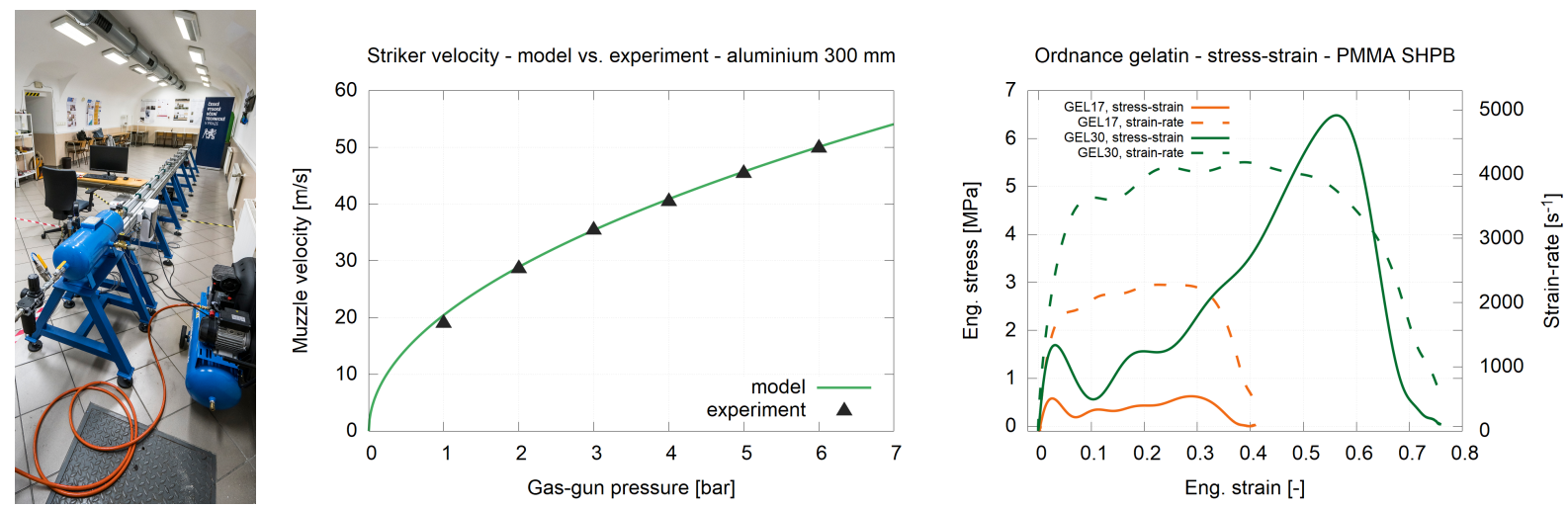

Fig. 2: Experimental setup (left), muzzle velocity - comparison of the model and the experiment (center), example stress-strain and strain-rate-strain graphs of the ordnance gelatin tested in SHPB with PMMA bars (right)

\subsection{Re-entrant auxetic lattice - aluminium bars}

An additively manufactured 2D re-entrant auxetic lattice (Fíla et al., 2017) (structure with negative Poisson's ratio) is presented here as an example of the model functionality for linear-elastic aluminium bars at two different impact velocities. It was experimentally proven that, in case of the aluminium bars, it is not necessary to take friction and pressure losses into account as the effects can be considered negligible in the used velocity range. Two different stresses $\sigma_{\mathrm{sp}}$ for the ideal plastic model of the specimen were used as strain-rate sensitivity effect was expected based on the previous results. Comparison of the results of the model and the experiment is summarized in Tab. 1. The values predicted by the model are in very good agreement with the measured values.

\subsection{Ordnance gelatin - PMMA bars}

A specimen of ordnance gelatin is presented in this paper as an example of the model functionality for visco-elastic PMMA bars at two different impact velocities. Damping and dispersion effects were corrected in the model using two different pressure losses coefficients $C_{\mathrm{P}}$ based on the wave propagation functions 
Tab. 1: Comparison of the experiment output estimated by the model with the values measured in the experiment - SHPB aluminium bars. Specimen - auxetic $2 D$ re-entrant. Model parameters: $\sigma_{\mathrm{sp}}=60 \mathrm{MPa}$ (low pressure), $\sigma_{\mathrm{sp}}=75 \mathrm{MPa}$ (high pressure), $C_{\mathrm{P}}=1, C_{\mathrm{f}}=0$.

\begin{tabular}{lcccccc}
\hline & \multicolumn{3}{c}{$P=1$ bar } & \multicolumn{3}{c}{$P=8$ bar } \\
\hline & model & experiment & relative diff. & model & experiment & relative diff. \\
\hline$v_{\mathrm{st}}[\mathrm{m} / \mathrm{s}]$ & 21.73 & 20.95 & $4 \%$ & 43.45 & 42.64 & $2 \%$ \\
\hline$\epsilon_{\mathrm{sp}}[-]$ & 0.28 & 0.27 & $4 \%$ & 0.61 & 0.52 & $17 \%$ \\
\hline$\dot{\epsilon}_{\mathrm{sp}}\left[\mathrm{s}^{-1}\right]$ & 1436 & 1374 & $5 \%$ & 3135 & 3015 & $4 \%$ \\
\hline$\epsilon_{\text {in }}[\mu \epsilon]$ & 2104 & 2122 & $1 \%$ & 4208 & 4311 & $2 \%$ \\
\hline
\end{tabular}

for PMMA. Two different stresses $\sigma_{\mathrm{sp}}$ for the ideal plastic model of the specimen were used as strain-rate sensitivity effect was expected based on the previous results. Comparison of the results of the model and the experiment is summarized in Tab. 2. The values predicted by the model are in very good agreement with the measured values. Measured stress-strain and strain-rate-strain curves are shown in Fig. 2-right.

Tab. 2: Comparison of the experiment output estimated by the model with the values measured in the experiment - SHPB PMMA bars. Specimen - ordnance gelatin. Model parameters: $\sigma_{\mathrm{sp}}=0.3 \mathrm{MPa}$ (low pressure), $\sigma_{\mathrm{sp}}=4 \mathrm{MPa}$ (high pressure), $C_{\mathrm{P}}=0.9$ (low pressure), $C_{\mathrm{P}}=0.8$ (high pressure), $C_{\mathrm{f}}=0$.

\begin{tabular}{lcccccc}
\hline & \multicolumn{3}{c}{$P=1$ bar } & \multicolumn{3}{c}{$P=8$ bar } \\
\hline & model & experiment & relative diff. & model & experiment & relative diff. \\
\hline$v_{\mathrm{st}}[\mathrm{m} / \mathrm{s}]$ & 11.11 & 11.72 & $5 \%$ & 21.91 & 24.51 & $11 \%$ \\
\hline$\epsilon_{\mathrm{sp}}[-]$ & 0.37 & 0.37 & $0 \%$ & 0.67 & 0.65 & $3 \%$ \\
\hline$\dot{\epsilon}_{\mathrm{sp}}\left[\mathrm{s}^{-1}\right]$ & 1985 & 2084 & $5 \%$ & 3767 & 3926 & $4 \%$ \\
\hline$\epsilon_{\text {in }}[\mu \epsilon]$ & 2585 & 2847 & $9 \%$ & 5097 & 5231 & $3 \%$ \\
\hline
\end{tabular}

\section{Conclusions}

A simple analytic model for prediction of the experiment output of a gas-gun propelled SHPB was derived from the adiabatic model of light gas-gun and from equations of the one-dimensional wave propagation theory in linear elastic slender bars. The model allows for calculation with elementary drag effects or for use of this part of the model for a simple correction of the wave dispersion effects in the measurement bars. The model functionality was evaluated on a real SHPB experiments carried out with both linear-elastic and visco-elastic bars at two different impact velocities. In all cases, the model predicted values close to the output of the real experiments. To conclude, the presented model can be used as a simple tool for prediction of the SHPB experiment output or inversely as a tool for designing a SHPB with required performance.

\section{Acknowledgments}

The research was supported by the Czech Science Foundation (project no. 15-15480S) and the internal grant of the Czech Technical University in Prague (project no. SGS17/148/OHK2/2T/16).

\section{References}

Fíla T. et al. (2017), Impact Testing of Polymer-filled Auxetics Using Split Hopkinson Pressure Bar. Advanced Engineering Materials, Vol. 19, No. 10, art. no. 1700076

Gallina F. , Birch R.S. and Alves, M. (2003) Design of a Split Hopkinson Pressure Bar. In: Proceedings of COBEM 2003, Sao Paulo, art. no. 1588

Rohrbach, Z. J., Buresh, T. R. and Madsen, M. J. (2011) Modeling the exit velocity of a compressed air cannon. American Journal of Physics, Vol. 80, No. 1, pp 24-26

Seigel A. E. (1981) Performance Calculations and Optimization of Gas Guns, US Army, Adelphi.

Stephenson, W. B. (1961) Theoretical light gas-gun performance, ASTIA, Arlington. 